\title{
Topology of music recommendation networks
}

\author{
Pedro Cano, a) Oscar Celma, and Markus Koppenberger \\ Music Technology Group, Universitat Pompeu Fabra, Ocata 1, 08003 Barcelona, Spain \\ Javier M. Buldúb) \\ Departament de Física i Enginyeria Nuclear, Universitat Politècnica de Catalunya, Colom 11, E-08222 \\ Terrassa, Spain
}

(Received 21 July 2005; accepted 21 October 2005; published online 12 January 2006)

\begin{abstract}
We study the topology of several music recommendation networks, which arise from relationships between artist, co-occurrence of songs in play lists or experts' recommendation. The analysis uncovers the emergence of complex network phenomena in these kinds of recommendation networks, built considering artists as nodes and their resemblance as links. We observe structural properties that provide some hints on navigation and possible optimizations on the design of music recommendation systems. Finally, the analysis derived from existing music knowledge sources provides a deeper understanding of the human music similarity perception. () 2006 American Institute of Physics. [DOI: 10.1063/1.2137622]
\end{abstract}

Music is ubiquitous in human societies. Music generates communities of musicians ${ }^{1,2}$ and communities of listeners. Nevertheless, the way music links people is certainly diverse and sometimes unexpected. In this work we focus on networks where musicians (or bands) are the fundamental nodes and are linked to others if they perform or compose similar music. This information is extracted from main online music recommendation systems: AllMusicGuide, MSN Entertainment, Amazon, and Launch Yahoo! Music recommendation systems are constructed to assist users to navigate through music collections, where navigation consists of guided links among artists. When the user selects an artist, a certain number of alternative artists are suggested, which in principle should be of his/her interest. In our study of the structure of different music recommendation systems we find characteristics that influence the systems' usability. Our results show that despite some common features, such as small worldness, different network characteristics exist, such as the link degree distribution. We show that there exists a relation between the link degree distribution and the construction of the networks. Networks constructed by collaborative efforts are scale free whereas networks with human experts supervising the links are exponential. This raises a discussion on the main forces driving the creation of the networks and hence their quality and potential uses. If preferential attachment takes place, as in the scale-free networks under study, the recommendations are biased toward popular items. On the other hand, exponential networks are more faithful to the underlying music similarity.

\section{INTRODUCTION}

Nowadays access to music is possible by querying artists or song names-editorial data—or browsing recommenda-

\footnotetext{
${ }^{\text {a) Electronic mail: pcano@iua.upf.es }}$

${ }^{b)}$ Electronic mail: javier.martin-buldu@upc.edu
}

tions generated by collaborative filtering-i.e., recommendation systems that exploit information such as "users that bought this album also bought this album." An obvious drawback is that consumers need to know the name of the song or the artist, or an important number of consumers must have heard and rated the music. This situation makes it difficult for users to access and navigate through the vast amount of music composed and performed by unknown new artists, which is available online in an increasing number of sites.

In this work, complex network measurements ${ }^{3,4}$ are used to analyze the topology of networks underlying the main music recommendation systems. The properties that emerge raise a discussion on the underlying forces driving collaborative systems and expert-guided networks. We can also obtain some hints about how much of the network structure is due to content similarity and how much to the selforganization of the network. Therefore, it can shed new light on the design and validation of music similarity measures and their evaluation. ${ }^{5}$ Further, it uncovers possible optimizations when designing music information systems, such as the optimal number of links between artists or the shortest path from artist to artist. In this sense, recommendation networks can be optimized by adding (or removing) links to facilitate navigating from artist to artist in a short number of clicks. Finally, we can obtain information about which artist has more links or which genres are more extended. This kind of information may help to understand the dynamics of certain aspects of music evolution, e.g., how did an artist get popular or how the music genres emerged.

\section{GRAPH DATASET}

We have gathered information from four different music recommendation networks: AllMusicGuide, ${ }^{6}$ Amazon, ${ }^{7}$ Launch-Yahoo!, ${ }^{8}$ and MSN Entertainment, ${ }^{9}$ and we have created a graph for each source, taking the "similarity" between artists as the linking parameter. A graph is constructed as 
TABLE I. Summary of the network parameters, where $n$ is the number or artists, $m$ is the number of links, $\langle k\rangle$ is the average degree, $C$ is the clustering coefficient, $C_{r}$ is the clustering of the equivalent random network, $d$ is the average shortest path, and $d_{r}$ is the corresponding shortest path for the random network. The last two columns correspond to the exponents of the power-law decay of the degree distribution for the incoming and outgoing links, $\gamma_{\text {in }}$, $\gamma_{\text {out }}$, of the graphs (we show exponents only when we found power-law decay).

\begin{tabular}{ccccccccccc}
\hline \hline Network & Type & $n$ & $m$ & $\langle k\rangle$ & $C$ & $C_{r}$ & $d$ & $d_{r}$ & $\gamma_{\text {in }}$ & $\gamma_{\text {out }}$ \\
\hline MSN & Directed & 51616 & 279240 & 5.5 & 0.54 & $1.0 \times 1.0^{-4}$ & 7.7 & 6.4 & $2.4 \pm 0.01$ \\
Amazon & Directed & 23566 & 158866 & 13.4 & 0.14 & $5.7 \times 10^{-4}$ & 4.2 & 3.9 & $2.3 \pm 0.02$ & $2.4 \pm 0.04$ \\
AMG & Directed & 29206 & 146882 & 8.15 & 0.20 & $2.8 \times 10^{-4}$ & 6.2 & 4.9 & - & - \\
Yahoo & Directed & 16302 & 511,539 & 62.8 & 0.38 & $3.8 \times 10^{-3}$ & 2.7 & 2.3 & - \\
\hline \hline
\end{tabular}

follows: each node represents a music artist whereas an edge denotes a similarity among them. The decision to create a link between two artists depends on the recommendation system's criterion, which can be different from network to network. Therefore, it is important to define how links between artists are created. The main characteristics of each network are summarized as follows:

- MSN Entertainment (MSN) is a portal to access multimedia content. Music can be accessed using editorial metadata, i.e., artist name or song title, as well as navigating through music styles. Another browsing feature, the SoundsLike Artists, allows users to navigate from artist to artist that sound similar. Ratings of similarity between artists are constructed from user contributions. MSN seems to follow a collaborative filtering approach ${ }^{10}$ to create links between artists.

- Amazon is an online retailer of music, it uses item-to-item collaborative filtering to recommend albums and artists, based on consumer ratings and habits. ${ }^{10,11}$ We study the network constructed by using similar artists' links. It is worth noting that in the Amazon network links are indirectly created by users whose knowledge of the network nodes is limited.

- AllMusicGuide (AMG) is a database of music content covering facts about albums and artists where several descriptions are considered, such as styles, moods, country of origin, even the birth date of the artist. An editorial group, made of a substantial number of music experts, is responsible for the addition of new nodes (artists) and their connections. Contributions from the users of the system are also accepted, but always under the supervision of the editorial group. In this sense, it is a network where a filtering process is applied. AMG defines different networks, e.g., influences, roots, performed songs by, and so on. In this work, in order to compare with other music recommendation networks, we focus in the network of similarity between artists.

- Launch Yahoo (Yahoo)! is a music entertainment portal that, among other features, allows users to navigate by similar artist. No information is given about how links between artists are created. Nevertheless, as we will see, some conclusions can be extracted from the analysis of the network properties.

As a general feature, all networks are directed, which means that an artist A (e.g., "Oasis") can be similar to an artist B (e.g., "The Beatles"), but not necessary in the oppo- site direction. The number of artists $(n)$ and links $(m)$ of each network is summarized in Table I.

\section{NETWORK PROPERTIES}

Before going into the data analysis, let us introduce some definitions and concepts that will be used in this article. A network or graph is a set of nodes (also called vertices) connected via links (also called edges). Networks connected by directed links are called directed networks whereas networks connected by undirected edges are called undirected networks. In order to make a decision about the network structure we have measured the following graph parameters:

- Degree: The degree $k_{i}$ of a vertex $i$ is the number of connections of that vertex and $\langle k\rangle$ is the average of $k_{i}$ over all the vertices of the network.

- Degree distribution: The degree distribution $P(k)$ is the proportion of nodes that have a degree $k$. The shape of the degree distribution can help to identify the type of network: regular networks have a constant distribution, since all nodes have the same amount of degrees, "random networks" "12,13 — as described by the Erdös-Rényi modelhave a Poisson degree distribution and "scale-free networks" have power-law distributions. ${ }^{20}$ It is a standard practice to compute the cumulative degree distribution $P_{c}(k)=\sum_{k^{\prime}>k} P\left(k^{\prime}\right)$ since it filters fluctuations of $P(k)$, which is frequently rather noisy. In a directed graph (all graphs under study) we can calculate $P^{\text {in }}(k)$ and $P^{\text {out }}(k)$ as the in and out degrees (for incoming/outgoing links), respectively.

- Average shortest path: A path between $i$ and $j$ exists if one can go from $i$ to $j$ following the edges in the graph. The path from $i$ to $j$ may not be unique. The minimum path distance or geodesic path $d_{i j}$ is the shortest path distance from $i$ to $j$. The average shortest path over every pair of vertices is

$\langle d\rangle=\frac{1}{\left.\frac{1}{2} n[n+1)\right]_{i \geqslant j}} d_{i j}$.

The maximum geodesic path between any two vertices in the graph is known as the diameter.

- Clustering coefficient: The clustering coefficient estimates the probability that two neighboring vertices of a given vertex are neighbors themselves. Concerning music recommendation networks, the clustering coefficient relates to 
the probability that if artist A is similar to artist $\mathrm{B}$ and artist $\mathrm{C}$, then artists B and C are similar as well. Following Ref. 14 the clustering coefficient of vertex $i$ is the ratio between the total number $y_{i}$ of the edges connecting its nearest neighbors and the total number of all possible edges between all these nearest neighbors. $c_{i}$ can be calculated following the expression (see Ref. 14 for details):

$c_{i}=\frac{2 y_{i}}{k_{i}\left(k_{i}-1\right)}$.

Finally, the clustering coefficient $C$ for the whole network is the average over the number of nodes $n$ :

$$
C=\frac{1}{n} \sum_{i} c_{i}
$$

Table I summarizes the network parameters of the different graphs, which will be analyzed in the following section.

\section{NETWORK STRUCTURE}

A common feature appears in all networks under study: they have small-world properties. ${ }^{14}$ The average shortest path $d$ (see Table I) of all graphs is below eight and always in the same order of magnitude as the shortest path of a random graph with the same number of nodes and links. This indicates that despite the high number of nodes (artists) and the sparsity of the network, a user can always jump from a node to any other by a short number of jumps (i.e., links). At the same time, the clustering coefficient $C$ is several orders of magnitude higher than that of the corresponding random network. Both ingredients, the low shortest path and the high clustering, are the typical characteristics of small-world networks. ${ }^{14}$ Small worldness is an interesting property for recommendation systems, since it has been suggested that humans find it easy to navigate in small-world networks using only local information. ${ }^{15-17}$

Concerning the average degree $\langle k\rangle$, we observe that it has a low value, in three of these systems (MSN, Amazon, and AMG) but it is higher in Yahoo $(\langle k\rangle=62.7)$. As we will discuss in the following section, the output degree tends to be bounded due to the usability constraints, i.e., the number of output links has to fit on a web page length.

The cumulative degree distribution $P_{c}(k)$ (distribution of nodes with a degree equal or higher than $k$ ) and specifically the way that $P_{c}(k)$ decreases allows to classify the smallworld networks. ${ }^{18}$ With this aim, we have analyzed the $P_{c}(k)$ distribution for all networks and we have found differences that are related with the internal structure and probably the construction mechanisms of each network.

Figure 1 shows the cumulative degree distribution of the (a) incoming and (b) outgoing links of the MSN recommendation network. It is worth noting the difference between both distributions. The incoming degree distribution $P_{c}^{\text {in }}(k)$ refers to artists who are similar to a selected artist. $P_{c}^{\text {in }}(k)$ is related with the number of links pointing to an artist and in a certain sense, it is an indicator of the influence of that artist over the others or how prototypical it is for a certain type of music. On the other hand, $P_{c}^{\text {out }}(k)$ refers to the number of
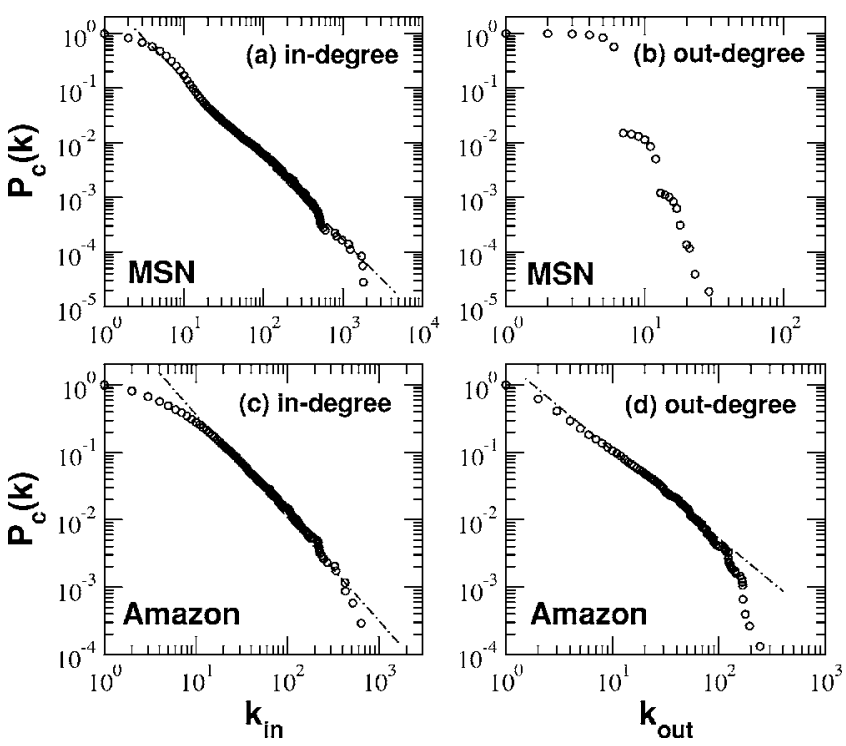

FIG. 1. $P_{c}(k)$ of MSN and Amazon recommendation networks (note the $\log$ - $\log$ scale in all plots). In the left column, the cumulative distributions $P_{c}^{\text {in }}(k)$ of the incoming degree $k_{\mathrm{in}}$. The cumulative distributions $P_{c}^{\text {out }}(k)$ of the outgoing degree $k_{\text {out }}$ are plotted in the right column.

output links from a given artists. The number of outgoing connections displayed to the user is limited for practical reasons since our networks underlie online systems where recommendations are shown on a web page, e.g., it would be useless and impractical to propose 2000 connections. This fact truncates the tail of the $P_{c}^{\text {out }}(k)$ distribution since not all the similar artists are linked and it reduces the expected value of $k_{\max }$ (Ref. 19) for scale-free networks.

$P_{c}^{\text {in }}(k)$ of MSN [see Fig. 1(a)] has a power-law decay $\left[P(k) \sim k^{-\gamma}\right]$, as indicated by the straight line in the log-log scale. Networks with a power-law decay are called "scale free" ${ }^{20}$ since we cannot identify a single characteristic scale. This kind of structure is common in small-world networks but not universal, and has been reported in different complex networks, such as the World Wide Web (WWW), ${ }^{21}$ the network of protein interactions, ${ }^{22}$ or the telephone call graph. ${ }^{23}$ The $P_{c}^{\text {out }}(k)$ distribution of MSN shows that the outgoing links are limited to 12 . Further, the fact that $P_{c}^{\text {out }}(k)$ drastically falls at $k=7$ reveals that each artist has typically six outgoing links. This limitation has very likely been introduced by the system designers. It levels the outgoing links of all nodes of the network and rules out any possibility of showing a power-law decay.

Figure 1 also shows the $P_{c}(k)$ of Amazon's recommendation network. $P_{c}^{\text {in }}(k)$ [Fig. $\left.1(\mathrm{c})\right]$ is quite similar to MSN, which indicates that both networks have a similar structure, at least for the incoming links. We find again a power-law decay, which indicates that Amazon is scale free. On the contrary, we find differences at $P_{c}^{\text {out }}(k)$, which keeps the power-law decay. This is not common on this kind of networks since it means that the outgoing links are not as limited as MSN; in fact there are nodes with more than 100 outgoing links [see Fig. 1(d)]. The absence of strong restrictions in the outgoing connections of the Amazon network allows both cumulative distributions (incoming/outgoing degree) to have similar shapes. 

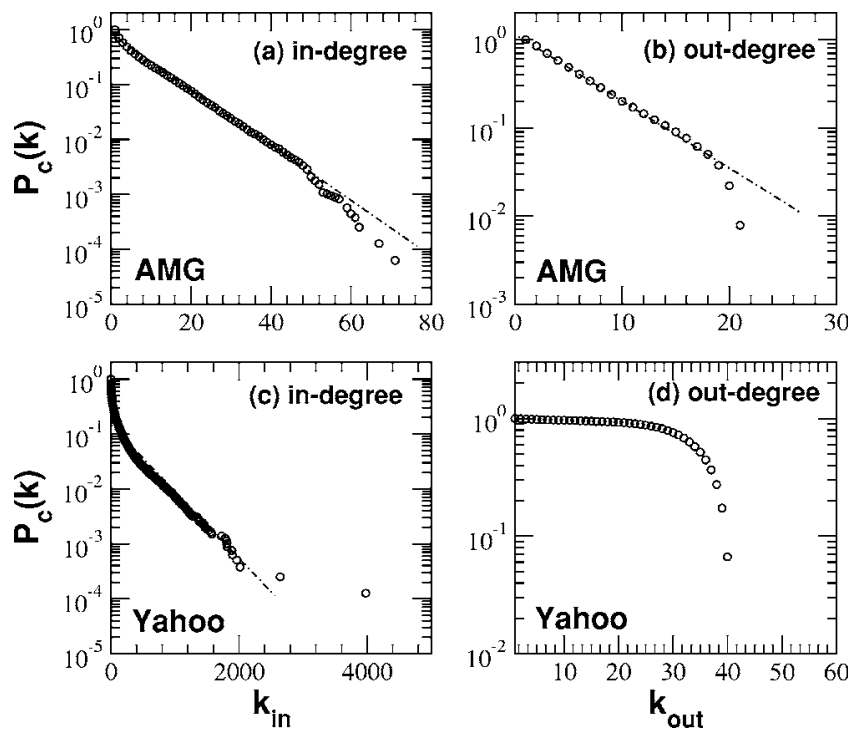

FIG. 2. $P_{c}(k)$ of AMG and Yahoo recommendation networks, in a linear-log scale. Left: the cumulative degree distributions $P_{c}^{\text {in }}(k)$ of the incoming links. Right: $P_{c}^{\text {out }}(k)$ of the outgoing links.

In Table I we have indicated the values of the power-law exponents $\gamma$ of Fig. 1 (see Ref. 24). In all cases, $\gamma$ is within the common range of values of previously studied scale-free networks. ${ }^{4}$

In Fig. 2 we have plot the cumulative degree distributions of the AMG and Yahoo networks, since both behave differently from the previous ones. The $P_{c}^{\text {in }}(k)$ distribution of AMG [Fig. 2(a)] has an exponential decay $\left[P(k) \sim e^{-k / \kappa}\right]$ since it follows a straight line in the linear-log scale (note the linear scale in the horizontal axes). $P_{c}^{\text {out }}(k)$ keeps the exponential decay [see Fig. 2(b)]. It is interesting to note that although the outgoing links are limited to $21, P_{c}^{\text {out }}(k)$ still has an exponential decay, although the slope is different from that of the incoming links.

Finally, we observe how Yahoo shows some similarities with the AMG network. Yahoo has an exponential decay for intermediate degrees of the $P_{c}^{\text {in }}(k)$ distribution [Fig. 2(c)], although it is lost for both low/high degrees. When looking at $P_{c}^{\text {out }}(k)$ [Fig. 2(d)], we see that the highest number of nodes is limited to 40 . Further, a typical number of $\sim 30$ outgoing links exists on each network, as we infer from the constant value (close to 1 ) of $P_{c}^{\text {out }}(k)$ from $k=1$ to $k \sim 30$. A similar characteristic was shown at the $P_{c}^{\text {out }}(k)$ of the MSN network [Fig. 2(d)] (in that case, outgoing links were set to $\left.k_{\text {out }}=5\right)$.

\section{DISCUSSION}

Some conclusions are drawn from the analysis of the networks' structure. Roughly speaking, we can say that we observe two types of network, one with power-law distribution of $P_{c}^{\text {in }}(k)$ and the second with an exponential decay. Since all networks are supposed to have the same functionality, i.e., recommendation of music based on artist similarities, it would be expected that all of them would have the same kind of structure. Nevertheless MSN and Amazon are scale free whereas Yahoo and AMG are exponential.

The reason for the structural differences could be explained by taking into account the ground characteristics of music networks together with their linking criteria. From the point of view of network categories, ${ }^{4}$ music networks can be considered as an information (or knowledge) network ${ }^{25}$ with a high social component. The structure of citations between academic papers is a classic example of an information network. ${ }^{26}$ In this sense, when an artist plays similar music to other artists, this is somehow "citing" their music. At the same time, a social component is unavoidable, since people are the elemental nodes of music networks. In both social and information network cases, a scale-free structure has been reported. ${ }^{4}$ This structure is associated with the preference of new nodes to associate with nodes with a high degree, i.e., with a high number of links. ${ }^{20}$

MSN and Amazon are user-rating- and user-habit-based networks. In both cases, links between artists are created by user ratings, buying behavior, or downloads statistics obtained from thousands of users in what is known as "collaborative filtering." 10 In such setups, each user inputs information on some of the nodes. Then all the information is aggregated and combined with the aim of predicting future ratings or, as explained in Ref. 10, to calculate the similarity between nodes. Of course, users have higher probabilities of linking artists (by rating or downloading) that they like. Since some artists are much more popular than others, they will get more links. From the obtained results, we find that this kind of collaborative filtering leads to a scale-free structure, at least for the case of music recommendation networks.

In order to check the hypothesis that popularity is behind the scale-free distribution, we use user behavior information from Art of the Mix. ${ }^{27}$ Art of the Mix (AOMix) is a website dedicated to sharing playlists submitted by a community of users. It contains almost 100000 playlists contributed by thousands of users. Playlist information from AOMix has been previously used by Refs. 5 and 29 as a source for music artist similarity. The underlying assumption considers that artists that co-occur in the same playlist are somehow similar. This is the idea behind "people who bought $X$ also bought $Y$," commonly exploited on online retailers such as Amazon. The properties of the network constructed adding a link between any two artists that coincide in a playlist are depicted in Table II. The cumulative degree distribution of AOMix is displayed in Fig. 3 and shows a power-law decay,

TABLE II. Summary of the network parameters for the Art of the Mix and the MusicSeer networks.

\begin{tabular}{ccccrcc}
\hline \hline Network & Type & $n$ & $m$ & $\langle k\rangle$ & \multirow{2}{*}{$C$} & $C_{r}$ \\
\hline Art of the Mix & Undirected & 48170 & 300708 & 12.5 & 0.1 & 0.003 \\
MusicSeer & Directed & 6144 & 10219 & 2.9 & 0.02 & $4.7 \times 10^{-4}$ \\
\hline \hline
\end{tabular}



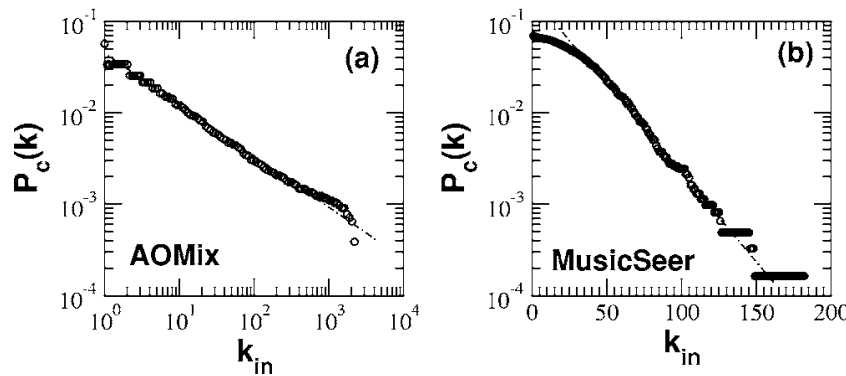

FIG. 3. $P_{c}(k)$ of AOMix (a) and MusicSeer (b) networks, in log-log and linear-log scales, respectively. Only the undirected distributions are displayed for ease of comparison (the AOMix graph is undirected).

as do MSN and Amazon. AOMix is originally a bipartite graph composed of playlist and artists nodes that has been projected into an artist node graph. It is worth mentioning that all networks built with collaborative-filtering algorithms derive from originally bipartite networks: e.g., People who listened to this $X$ also listened $Y$ or people who liked $X$ also liked $Y$. The use of what people do with or say about items, hence the exploitation of information of bipartite graphs, is the basis of collaborative filtering. ${ }^{10,11,25}$

Concerning the exponential scaling of Yahoo and AMG, we have no information about the wiring mechanisms of Yahoo (due to their privacy policy). Nevertheless, the AMG linking criterion is explained in detail in Ref. 6. This recommendation network is characterized by an editorial group of "experts" that supervises the wiring of the network. In this case, the construction of the network is uniquely guided by similarity criteria, a fact that cannot be guaranteed in the case of user-rating- or user-behavior-derived networks. In addition, it could be expected that human experts are in fact truncating possible scale-free structures by filtering links between normal artists and "hub" artists. ${ }^{28}$

Related to the exponential decay of Yahoo and AMG, it is worth commenting on another network used by Refs. 5 and 29 in the pursuit of a ground truth for music similarity. During a web experiment, named MusicSeer, users were asked to select the most similar artist to a given one from a list of ten possibilities. The properties of this human supervised network, where users have been explicitly asked to focus on similarity, is shown in Table II and its degree distribution is depicted in Fig. 3(b). In this case, as for Yahoo and AMG, an exponential degree distribution is obtained, and it is another example of how similarity music networks that try to avoid user preferences are prone to have exponential decay.

Both networks' degree distributions, AOMix and MusicSeer, are drawn next to each other to highlight how differences in the construction mechanisms affect the degree distribution. An exponential degree appears when similarity dominates over artist popularity; otherwise we observe power-law decays.

A crucial issue of the quality of recommendation networks is how searchable the networks are, that is, how easy is it for a user to find a target quickly. The influence of the network structure on the navigation has been addressed in the literature. ${ }^{15-17,30-32}$ Strategies for searching in scale-free networks using local information have been proposed by Refs. 30 and 31 . The algorithm selects the nodes with highest degree and scales sublinearly with the number of nodes. This type of algorithm cannot be exploited in the networks at hand. First, the power law is found for the in-degree distribution only. As we mentioned previously, the out-degree distribution has a cut due to web page usability constraints; the recommendation should fit on a web page. The in-degree distribution is unknown to the users, so it is unlikely that they choose the above proposed algorithm when searching in the recommendation networks. Second, it is unrealistic to think that users of the network can adopt such a search strategy, but rather the selection of nodes will be guided by their intrinsic qualities or some sort of underlying distance. ${ }^{16,17,32}$ Kleinsberg showed that lattices with random long-range links connected according to a distance-dependent probability distribution are searchable. ${ }^{15}$ Sublinear searches can be obtained assuming a small-word regime and the existence of a distance between nodes. ${ }^{17}$ Given its importance on the application, more work in this direction needs to be addressed. It would be very interesting to gather and analyze statistics of navigation of real users of the system.

\section{CONCLUSIONS}

We have analyzed the structure of music recommendation networks by means of complex network analysis. We have found small-world properties in all networks, which have an influence on the navigation properties of the network. Despite sharing the small-world structure, we have found differences in the scaling of their degree distribution. Networks with user preferences (from users of the network) as the linking criterion have a power-law decay of their degree distribution, i.e., show scale-free properties. On the other hand, networks constructed by similarity criteria lead to an exponential decay of the degree distribution. We believe that the scale-free and exponential decay could be related with the social or informational nature of the network. When the music recommendation network is constructed under the supervision of an editorial group, similarity aspects take advantage over the social ones and this is reflected in an exponential decay of the degree distribution. On the contrary, it is reasonable to expect that the social nature of the recommendation network increases in the case of user-preference linking. Finally, we give some insights about navigation through these kind of networks and address future work toward this point.

\section{ACKNOWLEDGMENTS}

The authors thank Juan A. Almendral, Fabien Gouyon, and Pablo de Miguel for fruitful discussions. Financial support was provided by MCyT-FEDER (Spain, Project Nos. BFM2002-04369 and BFM2003-07850), the Generalitat de Catalunya, and SIMAC IST-FP6-507142 European project.

${ }^{1}$ D. de Lima e Silva, M. Medeiros Soares, M. V. C. Henriques, M. T. Schivani Alves, S. G. de Aguilar, T. P. de Carvalho, G. Corso, and L. S. Lucena, "The complex network of the brazilian popular music," Physica A 332, 559 (2003).

${ }^{2}$ P. Gleiser and L. Danon, "Community structure in Jazz," Adv. Complex 
Syst. 6, 565 (2003).

${ }^{3}$ A.-L. Barabási, Linked: The New Science of Networks (Perseus, Cambridge, MA, 2002).

${ }^{4}$ M. E. J. Newman, "The structure and function of complex networks," SIAM Rev. 45, 167 (2003).

${ }^{5}$ D. P. Ellis, B. Withman, A. Berenzweig, and S. Lawrence, Proceedings of the International Symposium on Music Information Retrieval, Paris, France (2002), pp. 170-177.

${ }^{6} \mathrm{http} / / / \mathrm{www}$. allmusic.com

${ }^{7}$ http://www.amazon.com

${ }^{8}$ http://launch.yahoo.com

${ }^{9}$ http://music.msn.com

${ }^{10}$ B. Sarwar, G. Karypis, J. Konstan, and J. Riedl, Proceedings of the 10th International World Wide Web Conference, Hong Kong (2001), pp. 285295.

${ }^{11}$ G. Linden, B. Smith, and J. York, IEEE Internet Computing 4, 76 (2003).

${ }^{12}$ A. Rapoport, Bull. Math. Biophys. 10, 145 (1968).

${ }^{13}$ P. Erdös and A. Réyi, Publ. Math. (Debrecen) 6, 290 (1959).

${ }^{14}$ D. J. Watts and S. H. Strogatz, Nature (London) 393, 440 (1998).

${ }^{15}$ J. M. Kleinberg, Nature (London) 406, 845 (2000).

${ }^{16}$ D. J. Watts, P. S. Dodds, and M. E. J. Newman, Science 296, 1302 (2002).

${ }^{17}$ A. P. S. de Moura, A. E. Motter, and C. Grebogi, Phys. Rev. E 68, 036106 (2003).

${ }^{18}$ L. A. N. Amaral, A. Scala, M. Barthélémy, and H. E. Stanley, Proc. Natl. Acad. Sci. U.S.A. 97, 11149 (2000).
${ }^{19}$ The value of $k_{\max }$, which is the highest degree in the network, can be estimated in scale-free networks as $k_{\max } \sim n^{1 / \gamma-1}$.

${ }^{20}$ A. L. Barabási and R. Albert, Science 286, 509 (1999).

${ }^{21}$ A. Broder, R. Kumar, F. Maghoul, P. Raghavan, S. Rajagopalan, R. Sata, A. Tomkins, and J. Wiener, Comput. Netw. 33, 309 (2000).

${ }^{22}$ H. Jeong, S. Mason, A.-L. Barabási, and Z. N. Oltvai, Nature (London) 411, 41 (2001).

${ }^{23}$ J. Abello, A. Buchsbau, and J. A. Wesstbrook, Lect. Notes Comput. Sci. 1461, 332 (1998).

${ }^{24}$ Note that in this case we refer to degree distribution $P(k)$ instead of $\mathrm{cu}$ mulative degree distribution $P_{c}(k)$. Both power-law exponents are related by the expression $\gamma_{c}=\gamma-1$.

${ }^{25}$ S. Maslov and Y.-C. Zhang, Phys. Rev. Lett. 87, 248701 (2001).

${ }^{26}$ S. Render, Eur. Phys. J. B 4, 131 (1998).

${ }^{27}$ http://www.artofthemix.org

${ }^{28}$ S. Mossa, M. Barthélemy, H. E. Stanley, and L. A. N. Amaral, Phys. Rev. Lett. 88, 138701 (2002).

${ }^{29}$ A. Berenzweig, B. Logan, D. Ellis, and B. Whitman, Comput. Music J. 28, 63 (2004).

${ }^{30}$ B. J. Kim, C. N. Yoon, S. K. Han, and H. Jeong, Phys. Rev. E 65, 027103 (2002).

${ }^{31}$ L. A. Adamic, A. R. Puniyani, and B. A. Huberman, Phys. Rev. E 64, 046135 (2001).

${ }^{32}$ F. Menczer, Proc. Natl. Acad. Sci. U.S.A. 99, 14014 (2002). 